\title{
Visual search in cynomolgus monkeys: Stimulus parameters affecting two stages of visual search
}

\author{
MARK C. AZZATO and CHARLES M. BUTTER \\ University of Michigan, Ann Arbor, Michigan
}

\begin{abstract}
Monkeys searched for a target pattern presented at varying eccentricities (from 11 to 57 deg, along the horizontal meridian) with varying numbers of distractor patterns and at two contrast levels. The intercepts of the RT-target eccentricity functions were increasingly elevated as the number of distractors increased within the range presented in training, suggesting that increasing the number of distractors lengthened the time required to confirm the target's identity following each scanning phase of search. The additional increase of the intercepts when the number of distractors increased even further without benefit of training may be interpreted similarly. Decreasing the pattern contrast increased the slopes of the functions, as did increasing the number of distractors when low-contrast stimuli were presented, implying that these stimulus changes slowed the rate of scanning by increasing the number of fixations, their duration, or both. The slopes of the functions relating RTs to number of distractors (between 0 and 9) were .030 and $.035 \mathrm{sec} /$ distractor for the high- and low-contrast conditions, respectively.
\end{abstract}

Neisser (1967) has suggested that after prolonged practice visual search is accomplished in two stages. In the first stage, subjects scan a display (or list) rapidly, detecting the target by a preattentive process that operates on rather large parts of the display. They reported that when rapidly scanning a list of letters they do not "see" individual letters at all; everything is a blur from which the target letter "stands out" (Neisser, 1963). In the second stage, according to Neisser, subjects fixate and direct their attention to the target to which they were alerted in the scanning stage, so that it is identified.

Neisser's two-stage analysis of visual search raises the question: Do stimulus parameters that affect search time alter the array scanning rate (i.e., time per line in Neisser's studies), the target examination time, or both stages? Neisser (1963) has shown that several stimulus parameters-the target's form, its similarity to nontarget items, and the number of different targets searched foraffect unpracticed subjects' rate of array scanning, estimated by the slope of the search-time/target-position function. However, after extensive practice, the scan rate for several different targets, even for 10 of them (Neisser, Novick, \& Lazar, 1963), is as fast as the scan rate for a single target, although accuracy is somewhat reduced.

The authors thank C. C. Leiby for his assistance with the computer programs and Marylin K. Hoy for preparation of the manuscript. We also thank Henry Buchtel and Sylvan Kornblum for their criticisms and suggestions. The research reported in this paper was supported by a grant (MH 26489) from the National Institute of Mental Health. The authors' address is: Neuroscience Laboratory, University of Michigan, $1103 \mathrm{E}$. Huron Street, Ann Arbor, MI 48104-1687.
Another stimulus factor, the number of distractors in the display, has been shown in several studies (see Teichner \& Krebs, 1974) to affect the total search time of practiced subjects (for a different view of this effect, see Barber, 1981). However, it is not clear whether, for subjects with extensive search practice, increasing the number of distractors decreases the rate of array scanning or increases the time required to examine the target. Although extensive training has been found to abolish the initial effects of number of targets on rate of array scanning (Neisser et al., 1963), this may not be the case with number of distractors: Increasing the number of distractors placed randomly in a constant-size display increases their density and consequently reduces the effective field of view around the fixation point for a target (Mackworth, 1976), requiring subjects to scan a display at a slower rate. Thus, one might expect that the slope of the search-time/target-position function, the measure used by Neisser (1963) to estimate rate of display scanning, would increase as the number of distractors placed randomly in the display increased. If the slope of the function remained the same as the number of distractors increased, this would imply that the subjects were processing each distractor item faster or processing more than one item in parallel.

Alternatively (or in addition), increasing the number of irrelevant items might alter search time by lengthening the second stage of search. In this case, additional time would be added to search only once during each scan of the display-when the target was detected during the preattentive stage-assuming that a high rate of false detections did not occur during this stage. Consequently, the intercept of the search-time/target-position function would change as density varied. Of course, the 
intercept of the search-time/target-position function could also be affected by variations in the initiation of scanning or variations in the latency of the response by which the subject indicated target detection.

The present study was undertaken in order to determine whether varying the number of distractors presented in search training affected the intercept or slope (or both) of the search-time/target-position function in monkeys that had received extensive training in search for a target pattern. Monkeys, which had been tested in few visual search tasks (Collin, Cowey, Latto, \&. Marzi, 1982; Latto, 1978a, 1978b), were used as subjects in order to further study the generality across species of the effects on visual search of stimulus parameters examined extensively in humans. Furthermore, the use of monkeys in visual search tasks would make it possible to examine drug and brain-lesion effects on visual search, which might shed light on the brain mechanisms underlying search performance.

Another goal of this study was to determine which stage of visual search was affected by stimulus changes made without the benefit of extensive search training. One of the stimulus changes was increasing the number of distractors beyond those presented in training. Because, as mentioned above, increasing the number of distractors decreases the size of the effective field of view for a target (Mackworth, 1976), it was expected that this change in the search display might increase the slope of the search-time/target-position function. Similarly, it was also expected that decreasing the contrast of the target and distractors, another change that was made in the search task, might reduce the effective field of view for the target and consequently decrease the rate of scanning across the display, inasmuch as peripheral as well as foveal acuity declines as target contrast decreases (Daitch \& Green, 1969). The third stimulus change in the search task involved simultaneously increasing the number of distractors and decreasing the contrast of target and distractors.

The monkeys were trained and tested for visual search by methods similar to Latto's (1978b). On each trial, they (1) first responded to a small light (which required fixating within a few degrees of it) located between two display panels in order to present the stimuli on the display panels, and (2) then pressed the panel on which the target pattern was presented. Varying numbers of distractors (irrelevant patterns) were presented on both display panels, which were sufficiently large to require scanning within each in order to detect the target.

\section{METHOD}

\section{Subjects}

The subjects were eight adolescent cynomolgus monkeys (Macaca fascicularis), seven males and one female. They were housed in individual cages and fed daily with Purina Monkey Chow. The monkeys also received izoniazid tablets $(25 \mathrm{mg})$ daily as a prophylaxis against tuberculosis. Since they worked for water reward in the search task, their daily water intake during training and testing was restricted to $100-150 \mathrm{cc}$.

\section{Apparatus and Procedures}

The monkeys were trained and tested in a sound-shielded chamber painted flat black and dimly illuminated by an overhead lamp. The monkey, seated in a plastic restraining chair, faced two 24- $\mathrm{cm}^{2}$ stimulus-response panels constructed of frosted Plexiglas and referred to as the choice response (CR) panels. The CR panels were hinged at the top, so that a microswitch closed when they were pressed. A small $\left(3.5-\mathrm{cm}^{2}\right)$ panel, referred to as the OR (orienting response) panel, was located between the two CR panels and illuminated from the rear by a 28-V lamp (see Figure 1). Depression of this panel also closed a microswitch. When an electrically operated shutter opened, a slide was projected upon the CR panels. A solenoid valve delivered $.3-.5 \mathrm{cc}$ water through a curved metal tube mounted below the OR panel and terminated $18 \mathrm{~cm}$ in front of it. Since the monkeys kept their mouths in contact with the tip of the tube, they faced the panels and kept their heads in the center of the display area. A microprocessor (AIM-65, Rockwell, Int.) analyzed choice response latencies and reinforcements off-line.

\section{Initial Training}

After learning to press the CR panels for water reward, the monkeys were trained to press, for water reward, only the one CR panel that was illuminated with diffuse light on each trial. In each training session of 128 trials, the order in which the CR panels were illuminated was randomized. The intertrial interval was $5 \mathrm{sec}$. After performing $90 \%$ correct responses in two consecutive sessions, the monkeys were trained to press the OR panel only when it was illuminated with a light spot, in order to initiate a trial. The time during which the OR panel was illuminated was then gradually reduced to $3 \mathrm{sec}$. Next, the spot was reduced in size and brightness so that its detection by human observers required fixating within $2-3 \mathrm{deg}$ of it. The OR light came on 3-8 sec after termination of the previous trial. A response to the OR panel or to a CR panel at inappropriate times initiated a time-out period of 6-11 sec, during which a trial could not be initiated. After the monkeys learned to respond correctly to the OR light, the area illuminated on the CR panel was gradually reduced to a small disk of light, $0.9 \mathrm{~cm}$ in diameter. At the viewing distance employed (approximately $18 \mathrm{~cm}$ ), the

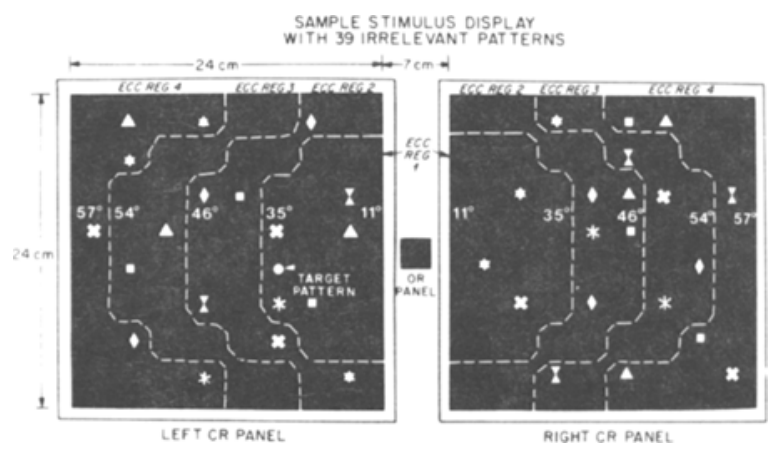

Figure 1. Diagrammatic representation of the display panels used in training and testing, drawn to scale, with 39 irrelevant patterns, 20 on the right CR panel and 19 on the left CR panel, which also contains the target pattern. The eccentricity regions (ECC REG), whose borders are indicated by the dashed lines (not displayed to the subjects), are approximately equal in area. The borders of the eccentricity regions were constructed in accordance with lines dividing each panel into an $8 \times 8$ matrix. 
diameter of the disk was approximtely 2.9 deg when it was located near the inner edge of the panels and approximately 1.9 deg when it was located near the outer edges of the panels. The monkeys were trained until they responded to the CR panel on which the disk was presented on at least $90 \%$ of the 128 trials in each of three consecutive sessions.

\section{Visual Search Training}

In this stage of training, the monkeys received practice in a task in which they were required to search for a target pattern presented with varying numbers of distractors, all of which were presented at high contrast. Visual search training differed in two major respects from initial training: (1) the position of the target pattern (the disk) within each CR panel was systematically varied, and (2) varying numbers of distractor patterns were presented on both CR panels. The distractors consisted of seven different forms-a square, diamond, cross, asterisk, triangle, and star, and a form composed of two triangles with the apexes touching (see Figure 1). The target and distractors were photographed, and slides displaying them were projected on the panels by a Kodak Carousel projector. In the first series of training sessions, the target pattern was presented alone ( 0 distractors). Its order of appearance on the left and right $C R$ panels was determined by a Gellermann series. In each training session of 128 trials, the monkeys were presented with the target pattern once in each of 128 positions obtained by dividing each CR panel into an $8 \times 8$ matrix. The order in which the target pattern was presented in the 64 positions within each panel was varied randomly. The brightness of the target pattern was $13.1 \mathrm{~cd} / \mathrm{m}^{2}$; the illumination inside the apparatus reflected $1.27 \mathrm{~cd} / \mathrm{m}^{2}$ on the panels. Choice response latencies (from the time of the onset of the electric signal opening the shutter to the time when the monkey depressed one of the CR panels) were recorded by the microprocessor. The monkeys were tested with the target pattern alone until they achieved $90 \%$ correct responses in each of three consecutive sessions. They were next trained with successively more distractors-first 1 , then $3,5,7$, and finally 9 , the positions of which in the $8 \times 8$ matrix within each CR panel were randomized. The form of the distractor at each position was chosen randomly with the restriction that each of the seven forms appeared no more than once in each trial when 1-7 distractors were presented. The brightness of the distractors was the same as that of the target. On each trial, the total number of patterns (including the target pattern) projected on each $C R$ panel was the same. The monkeys were required to achieve $90 \%$ correct responses in each of three consecutive sessions with each number of distractors. In the last training session with nine distractors, the animals were also required to respond with median RTs equal to or less than the mean of the median RTs on the last four sessions. Two of the eight monkeys were also required to meet this criterion when they were trained with 0-7 distractors. In all other respects, the procedures were the same as those used in training with 0 distractors.

\section{Visual Search Testing}

After completing visual search training, the monkeys were tested in four series of visual search tests. In the first series, referred to as the $0-9$ high-contrast series, the monkeys were tested with patterns of the same number and contrast presented in training. They were presented in each sesison with displays consisting of the target pattern and $0,1,3,5,7$, and 9 distractors, all presented at the same brightness level used in visual search training. The shape of the distractors and their positions within each CR panel were randomized. In this series, as in the subsequent ones, the monkeys were rewarded only for pressing the panel on which the target pattern was presented. Each session consisted of six blocks of 32 trials each; within each block, the number of distractors was constant. The order of presentation of the six blocks was randomized in each session. The monkeys were tested in the 0-9 high-contrast series until they had completed eight sessions in which they had performed with at least $90 \%$ correct responses and in which they had responded at least $90 \%$ of the time to each onset of the OR light. In these eight sessions, the target pattern appeared an equal number of times in each of the 64 positions on each CR panel and 32 times in each of the four eccentricity regions in each panel (see Figure 1).

The monkeys were next tested in a series of sessions ( 39 highcontrast series) in which they were presented on each trial with 39 distractors, which, together with the target pattern, were all high-contrast $\left(13.1 \mathrm{~cd} / \mathrm{m}^{2}\right)$. Nineteen distractors were presented on the CR panel containing the target pattern; the remaining 20 distractors were presented on the other panel. The forms of the distractors and their positions within the panel were randomized on each trial. Each session consisted of 128 trials. The monkeys were tested in this series until they had performed with at least $90 \%$ correct responses in four sessions, during which the target pattern appeared an equal number of times in each of the 64 positions on each panel and 64 times in each of the four eccentricity regions in each panel. The monkeys were next tested in a series of sessions, referred to as the 0-9 lowcontrast series, in which the procedures were the same as in the previous $0-9$ series, except that the luminance of all the patterns was reduced to $1.31 \mathrm{~cd} / \mathrm{m}^{2}$, while the background luminance $\left(1.27 \mathrm{~cd} / \mathrm{m}^{2}\right)$ remained the same as it had been when the highcontrast patterns had been presented. Finally, the monkeys were tested in a series of sessions, referred to as the 39 low-contrast series, which differed from the 39 high-contrast series only in that the luminance of the patterns was reduced to $1.31 \mathrm{~cd} / \mathrm{m}^{2}$. In all other respects, the procedures used in the four series of tests were the same as those used in the final stage of visual search training.

\section{RESULTS}

All statistical analyses of RTs reported here were performed only on RTs of correct choice responses in test sessions.

The monkeys required 33-59 sessions of search training. In testing sessions in which the stimulus parameters were the same as in training (0-9 high-contrast series), the median RTs of choice responses (1) increased as the target pattern was presented more eccentrically on the CR panels, and (2) were longer when the target pattern appeared on one CR panel (the left panel for half of the subjects and the right panel for the others) than they were when the target appeared on the other CR panel. The same pattern of RT changes was found when the monkeys were tested with 39 distractors at high contrast. In order to more clearly display and analyze these patterns of $R T$ variation, the CR panel on which all the monkeys responded with shorter RTs was arbitrarily designated the left $C R$ panel and the CR panel on which they responded with longer RTs was designated the right $C R$ panel. The rearranged median RTs of correct responses, averaged over subjects, are plotted in Figure 2 against the eccentricity region (see Figure 1) in which the target pattern appeared, with number of distractors as the parameter.

It is clear from inspection of Figure 2 that median RTs in the stimulus conditions used in training (0-9 highcontrast series) changed systematically as the position of the target pattern on the panels varied; moreover, these 


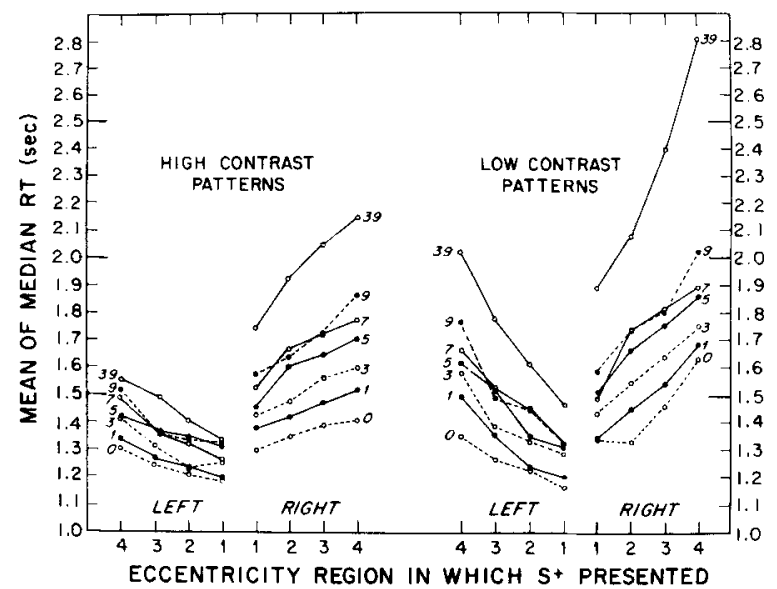

Figure 2. Average median RTs of correct choice responses in search tests with 0-9 high-contrast distractors and 39 highcontrast distractors (left side), and 0-9 low-contrast distractors and 39 low-contrast distractors (right side), all plotted against the eccentricity region in which the target pattern (S+) appeared. The centers of the eccentricity regions in the CR panels were approximately equidistant from each other.

RT changes occurred at all levels of distractor number (see left side of Figure 2). This effect of target position was reflected in a two-way ANOVA of median RTs in the 0-9 high-contrast tests as a highly significant main effect of eccentricity region in which the target was located $[F(7,366)=20.86, p<.001]$. Furthermore, it is evident from inspection of Figure 2 that median RTs in the $0-9$ high-contrast tests became longer as the number of distractors increased, a main effect that was also highly significant $[F(5,337)=16.86, p<.001]$. Although the intercepts of the RT-target eccentricity functions were increased as the number of distractors increased from 0 to 9 , their slopes were not reliably steepened: The interaction between target eccentricity and number of distractors was not significant $[F(35,337)$ $=.65]$.

When the high-contrast display with 39 distractors was presented, the RT-target eccentricity functions not only showed increased intercepts, but also became steeper, especially when the target was presented on the second (right) panel (see Figure 2). However, when RT data collected with 39 distractors were included in an ANOVA, the interaction between target eccentricity and number of distractors was still not significant $[F(42,392)$ $=0.93]$. Nevertheless, since the findings in Figure 2 suggest that in the high-contrast tests the number of distractors did affect RT changes with target eccentricity, the data were further analyzed by orthogonal comparisons (Winer, 1962). Orthogonal comparisons were made of differences between median RTs of responses to target presentation in eccentricity region 1 and median RTs of responses to target presentation in eccentricity region 4 of the same CR panel at each level of distractor number. Only one comparison was statistically significant: the difference between median RTs when the target was located in eccentricity regions 1 and 4 of the right panel was significantly larger with 39 distractors than was the comparable RT difference with 0 distractors $[\mathrm{F}(1, \infty)=$ $4.21, \mathrm{p}<.05]$.

Turning to the results of visual search tests with lowcontrast stimuli (see right side of Figure 2), the median RTs increased systematically as the eccentricity of the target varied at all levels of distractor number, as in the high-contrast tests. An ANOVA of all the low-contrast data revealed that this effect of target eccentricity was highly significant $[F(7,392)=64.09, p<.001]$. It is also clear that RTs systematically increased as number of distractors increased, an effect that was also highly significant $[F(6,392)=78.80, p<.001]$. Furthermore, it appears that the RT-target eccentricity functions in the low-contrast condition did not systematically change in slope as number of distractors increased from 0 to 9 , but did become steeper when 39 distractors were presented. This conclusion is supported by the results of two ANOVAs: When the RT data from tests with 39 distractors were excluded from the analysis, the interaction between target eccentricity and number of distractors did not attain significance $[F(35,336)=0.66]$. However, the interaction was significant when RT data from tests with 39 distractors were included in the analysis $[\mathrm{F}(42,392)=2.32, \mathrm{p}<.001]$.

The contrast level of the patterns also affected RTs in the search tests; across all four tests, RTs were significantly longer when the patterns were low in contrast than when the same patterns were high in contrast $[F(1,819)=33.6, p<.001]$. Furthermore, it appears, as indicated in Figure 2, that when low-contrast patterns were presented, the intercepts of the RT-target eccentricity functions between 0 and 9 distractors did not increase, but the functions were steeper than they were when high-contrast patterns were presented. This effect resulted in a significant interaction between contrast level and target eccentricity $[F(7,701)=6.17, p<.001]$. However, contrast level did not interact significantly with number of distractors $[F(5,701)=0.56]$ when they varied between 0 and 9 .

In order to examine in greater detail the effect of number of distractors on RTs, the median RTs at each level of pattern contrast were fitted by the least squares method to separate functions for $0-9$ and $0-39$ distractors (see Figure 3). The regression lines fitted to the functions between 0 and 9 distractors appear quite linear, although the RT increments from 0 distractors (a detection task) to 1 distractor (a discrimination task) were larger than average, and the RT increments from 7 to 9 distractors were smaller than average. However, the nonlinear components in both the high-and low-contrast functions between 0 and 9 distractors were not statistically significant [for high contrast, $F(4,42)=.379$; for low contrast, $F(4,42)=.398$ ]. In contrast, the linear components in both of these functions were highly significant [for high contrast, $F(1,42)=8.64$, 


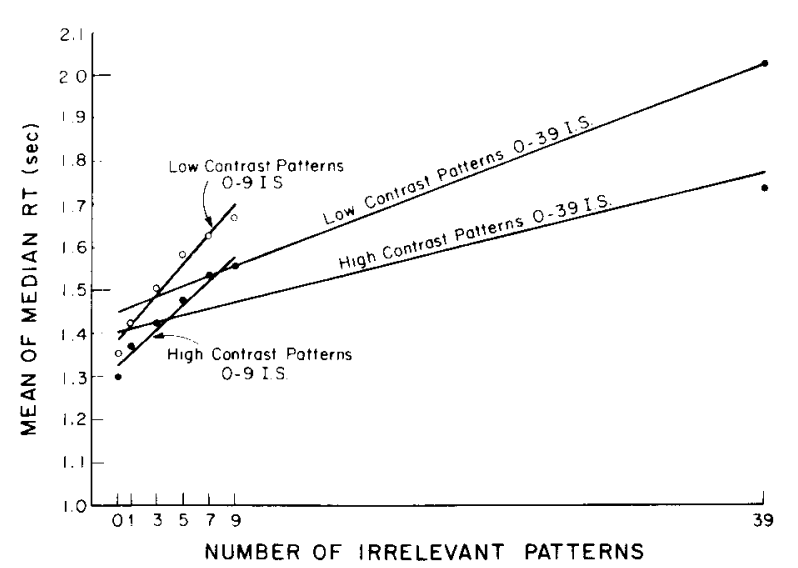

Figure 3. Average median RTs of correct responses plotted as a function of number of distractors, with pattern contrast as the parameter. Functions for the two ranges of distractor number (0-9 I.S. and 0-39 I.S.) at the two contrast levels were fitted by the least squares method.

$\mathrm{p}<.01$; for low contrast, $\mathrm{F}(1,42)=7.69, \mathrm{p}<.01]$. Furthermore, it is obvious that the slopes of the functions including 39 distractors were much lower than the slopes of the functions from $0-9$ distractors. Furthermore, the slopes of the RT functions between 0 and 9 distractors are quite similar to each other (see Figure 3); they were .030 and $.035 \mathrm{sec} /$ distractor for high- and lowcontrast stimuli, respectively, and the confidence intervals about them overlapped. Moreover, the slopes of the regression functions for individual subjects' data showed remarkably little variation: for high-contrast patterns, the range was $.016-.036 \mathrm{sec} /$ distractor; for low-contrast patterns, the range was $.023-05 \mathrm{sec} /$ distractor. The three-way interaction between target position, number of distractors, and degree of contrast was not significant.

Since the monkeys made very few choice errors during test sessions (less than 10\%), these errors could not be analyzed statistically. Of the few errors committed, most occurred when the target pattern was presented in the two most eccentric regions of the CR panels and 7-39 distractors were also presented (see Table 1).

The manner in which RTs changed as the position of high-contrast targets changed suggests that in the test series with high-contrast patterns the monkeys first scanned one CR panel and then the other from their innermost to outermost eccentricity regions. The top half of Figure 4 diagrammatically shows this search strategy, referred to as search strategy $A$, along with median RTs predicted by it. When the patterns were of low contrast, the relationship between RT and target eccentricity changed in the following ways: (1) The RT increment between eccentricity regions 3 and 4 of the first (left) CR panel scanned was larger than it was when high-contrast patterns were presented, and (2) the average RTs to the target presented in the two innermost eccentricity regions of the panel scanned second (right) overlapped the average RTs to the target presented in the outermost eccentricity region of the panel scanned first (see lower panel of Figure 4). These findings suggest that when low-contrast patterns were presented, the monkeys used search strategy A on some trials, but on the others they scanned all or most of the second panel before scanning the outermost eccentricity region of the first panel, and then finished scanning the second panel, if they had not already done so. The lower half of Figure 4 shows this strategy, referred to as search strategy B, together with strategy A and RTs

Table 1

Mean Percent Errors Committed in Search Testing

\begin{tabular}{|c|c|c|c|c|c|c|c|c|}
\hline \multirow{3}{*}{$\begin{array}{l}\text { Number of } \\
\text { Distractors }\end{array}$} & \multicolumn{8}{|c|}{ Target Position } \\
\hline & \multicolumn{4}{|c|}{ Left CR Panel } & \multicolumn{4}{|c|}{ Right CR Panel } \\
\hline & 4 & 3 & 2 & 1 & 1 & 2 & 3 & 4 \\
\hline & \multicolumn{8}{|c|}{ High Contrast } \\
\hline 0 & 1.1 & 0.5 & 1.1 & 0.0 & 0.0 & 0.0 & 0.5 & 1.2 \\
\hline 1 & 0.9 & 0.9 & 1.2 & 0.9 & 0.5 & 0.6 & 1.6 & 1.6 \\
\hline 3 & 0.7 & 4.2 & 0.6 & 0.5 & 0.3 & 1.0 & 0.4 & 2.0 \\
\hline 5 & 1.6 & 0.5 & 2.1 & 0.0 & 0.5 & 0.6 & 0.2 & 3.0 \\
\hline 7 & 3.9 & 1.9 & 0.5 & 2.6 & 0.3 & 2.0 & 0.5 & 2.7 \\
\hline 9 & 4.1 & 1.1 & 0.5 & 0.5 & 1.5 & 1.6 & 1.2 & 2.9 \\
\hline \multirow[t]{2}{*}{39} & 4.6 & 3.7 & 1.3 & 1.3 & 1.1 & 3.6 & 1.8 & 1.1 \\
\hline & \multicolumn{8}{|c|}{ Low Contrast } \\
\hline 0 & 1.6 & 1.0 & 0.6 & 1.0 & 0.5 & 0.0 & 0.5 & 0.0 \\
\hline 1 & 2.6 & 2.6 & 0.5 & 1.6 & 0.5 & 1.0 & 2.1 & 2.1 \\
\hline 3 & 2.4 & 2.6 & 0.8 & 4.2 & 1.6 & 0.6 & 0.2 & 1.6 \\
\hline 5 & 5.3 & 3.7 & 2.6 & 0.5 & 1.1 & 1.1 & 1.0 & 2.6 \\
\hline 7 & 6.8 & 3.6 & 0.5 & 0.5 & 2.1 & 1.2 & 2.6 & 4.7 \\
\hline 9 & 5.7 & 2.6 & 1.0 & 1.0 & 1.6 & 3.1 & 3.6 & 6.8 \\
\hline 39 & 5.7 & 3.8 & 1.6 & 0.9 & 1.5 & 1.2 & 1.6 & 8.9 \\
\hline
\end{tabular}

Note-Left $C R$ panel refers to the panel on which the subjects responded with shorter RTs; right CR panel refers to the panel on which the subjects responded with longer $R T$ s. 

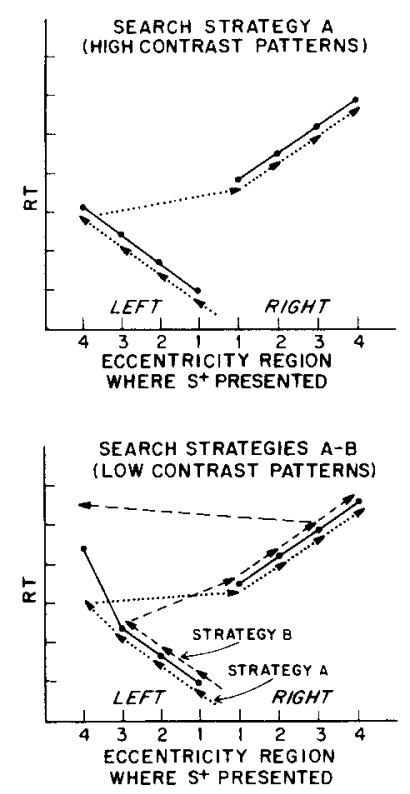

Figure 4. Diagrammatic representations of search strategies used in testing. Top: Search strategy $A$ (dotted line and arrowheads) together with typical pattern of RTs (solid line, filled circles) in tests with high-contrast patterns. Bottom: Search strategies $A$ and $B$ (both shown by dotted lines and arrowheads) together with typical pattern of RTs (solid line, filled circles) in tests with low-contrast patterns.

resulting from use of each strategy on $50 \%$ of the trials. The combination of these two strategies is referred to as the compound strategy A-B.

\section{DISCUSSION}

The modifications we made in the procedures employed by Latto (1978b) -the use of a restraining chair, the centered water spout, and the small light spot to which the monkeys oriented before each trial-prevented gross movement and required a monkey to center its head and eyes at the beginning of each trial, as done in many human studies of visual search. These modifications probably reduced the variability of RTs between subjects and, more importantly, facilitated the use of a uniform search strategy in training, as shown by the systematic and linear variations in RT with target eccentricity found in the first series of tests $(0-9$ highcontrast series). It also appears that the monkeys transferred this strategy to the tests in which the number of high-contrast distractors was increased to 39 . Evidence for systematic eye movements of subjects searching for a target in a visual display containing irrelevant items has been reported in several human studies (Gould, 1973; Mackworth, 1976; Williams, 1966).

An alternative explanation of the systematic increases in RTs with target eccentricity assumes that the subjects reached directly to the target in making their choice responses, as monkeys do when the discriminative stimuli are spatially displaced from the response sites (Polidora \& Fletcher, 1964). It might then take them longer to reach to an eccentrically located target than to one located near the center of the display. However, observations of the monkeys during testing revealed that only one of them consistently reached to the area of the panel on which the target was projected. Moreover, this monkey's RT-target eccentricity function was not steeper than the average function of the others.

Another interpretation of the RT increases with target eccentricity is based on the assumption that the monkeys fixated each panel only once and processed all the patterns on each panel in parallel. If this were the case, the fact that RTs were longer to peripheral than to central targets may have been due to the slower processing that would result from the decreased retinal size of the peripheral target (the viewing distance being greater to the periphery than to the center of the screen) and possibly from its slight distortion in projected retinal shape. However, it seems unlikely that the monkeys could process all, or even most, of the patterns in a single fixation on each panel; at the viewing distance employed, the screen was approximately $57 \mathrm{deg}$ wide, whereas the effective field of view for target identification, estimated by human observers, was no more than 20 deg wide.

It appears that the monkeys' search pattern-scanning first across one panel and then across the other-was only slightly altered when the contrast of the patterns was reduced, whether the number of distractors remained the same as in training or was increased to 39. The apparent change in the monkey's search strategy on some trials-scanning the second panel before completing the scanning of the first panel-might have been due to monkeys' limiting their head movements and occasional failure to move their eyes far enough to identify eccentrically located, low-contrast patterns on some trials. The somewhat smaller retinal size of the eccentrically placed patterns may have also made them more difficult to identify. The somewhat greater frequency of errors in choice responses to low-contrast eccentrically placed targets than to high-contrast eccentrically placed targets supports this interpretation.

The finding that the slopes of the RT-target eccentricity functions did not change significantly as the number of distractors increased in the tests with the $0-9$ high-contrast series suggests that the monkeys' scanning rate across the display did not significantly change under these testing conditions. Furthermore, since the slope of the function was not significantly altered, even when 39 high-contrast distractors were presented, it appears that the monkeys' scanning rate remained constant when the number of distractors was increased beyond the number used in training. This interpretation implies that as the number of distractors increased, the subjects were processing each distractor faster or using parallel processing. Estimation by direct inspection of the slopes of the functions obtained in the 0-9 high-contrast tests suggests 
that the monkeys scanned from the OR panel to the far edge of each choice panel, a distance approximately $57 \mathrm{deg}$ in horizontal extent, in $200 \mathrm{msec}$ or less. Since the effective field of view for the target was no more than $20 \mathrm{deg}$ wide for human observers, the monkeys, when scanning laterally, probably made two or three fixations to reach a target at the far edge of a panel and place it in their effective field of view. Since, as mentioned above, it appears that the monkeys scanned this distance in no more than $200 \mathrm{msec}$, each fixation in the 0.9 high-contrast tests would have to be quite short60-100 msec.

Although the slopes of the RT-target eccentricity functions obtained in the high-contrast tests did not change reliably, their intercepts increased as the number of distractors increased. Blough (1979), using pigeons as subjects, also found that as the number of irrelevant stimuli to which the pigeons had been exposed in search training increased, the intercept of the RT/target-position function was raised; however, it is not clear from these findings whether the slope of the function was also changed (D. Blough, personal communication, 1982). Furthermore, in the 0-9 high-contrast tests, RTs increased in a linear manner with number of distractors, at an average rate of $30 \mathrm{msec} /$ distractor, with little variation among individual subjects. These findings suggest that increasing the number of distractors affected the second, or checking, stage of search, adding approximately $30 \mathrm{msec}$ to it for each addiitonal distractor between 0 and 9 . Since, as mentioned above, fixation durations in search testing were estimated to be 60 $100 \mathrm{msec}$, it would appear that in the $0-9$ high-contrast tests, the monkeys were processing two or three distractors in each fixation. Furthermore, assuming that fixations during the checking stage of search with 0 distractors, as in the scanning stage, were not shorter than $60 \mathrm{msec}$, and that $30 \mathrm{msec}$ was added for each distractor, as more distractors were added to the display, the duration of the checking time would have been longer than fixation durations during the scanning phase. This conclusion is consistent with Gould's (1973) finding that the duration of fixations on targets is longer than the duration of fixations on nontargets in human search.

The finding that the RT increment from 9 to 39 distractors was much smaller than would be predicted from the function relating RT to 0-9 distractors suggests that the monkeys were processing the displays with 39 distractors at an even faster rate than they processed the displays with fewer distractors. It is possible that this faster rate of processing was due to a difference in search testing procedures; the tests with sparse displays, unlike the tests with 39 distractors, involved several numbers of distractors.

The RT increments with increasing number of distractors may have been due to an increase in duration of pauses in initiating scanning or to increased response time following the checking stage. However, it is un- likely that the monkeys increasingly delayed search initiation as the number of distractors increased, because the small size of their estimated effective field of view would limit the information they could obtain from the greater part of the choice panels while they were looking at the OR panel. The possibility that the RT increment with increasing distractor density was due to response factors cannot be ruled out.

It is also possible that increasing the number of distrators elevated RTs in the search task by increasing the density of the distractor patterns. It has been shown in human studies that density increases RT in letterrecognition (Estes, 1972; Strangert \& Branstrom, 1975) and visual-search (Eriksen \& Hoffman, 1972; Treisman, 1982) tasks. However, in the human studies, the highdensity stimuli were separated by less than $1 \mathrm{deg}$, whereas, in the present study, the patterns that occupied adjacent positions were no less than $4 \mathrm{deg}$ apart. According to Collins and Eriksen (1967), the masking effect of adjacent patterns on letter recognition requires stimulus separations of less than $1 \mathrm{deg}$. Furthermore, Treisman (1982) has reported that although increasing the number of distractors independently of density produces a large effect on RT, changing their density produces a relatively small effect. For these reasons, then, it is unlikely that density produced a significant effect on RTs in the present experiment.

The effect of reducing the pattern contrast was to steepen the slopes of the RT-target eccentricity functions between 0 and 9 distractors, without increasing their intercepts. According to Neisser's (1967) twostage theory of search, this finding suggests that when the task was made more difficult by reducing stimulus contrast, the subjects took longer to complete the preattentive scanning phase but not the checking phase. When the search task was made even more difficult, by increasing the low-contrast distractors to 39 , the functions not only became steeper than they had been when the number of low-contrast distractors was smaller, but they also showed increased intercepts. Thus, it appears that increasing the number of low-contrast distractors to 39 lengthened not only the duration of the checking time but also the duration of the scanning phase of search. It should be pointed out that reducing the stimulus contrast might have led to larger changes in the slopes of the RT-target eccentricity functions, or might have affected other aspects of search performance, had the order of the high-and low-contrast conditions been counterbalanced.

Decreasing contrast or increasing the number of lowcontrast distractors could lengthen the scanning phase by (1) increasing fixation durations, (2) increasing the number of eye movements, or (3) increasing the duration of eye movements. Changes in the velocity of eye movements in visual search have not been reported. With regard to the other two possibilities, Luria and Strauss (1975) found that the efficiency of search was more closely related to the number of fixations than 
it was to fixation durations. Of greater relevance to the present results is the finding that the additional time required by well-practiced subjects to detect a target when a complex background is added to the task is due primarily to the increase in number of fixations (Gould \& Cam, 1973). This is a finding that might be expected, inasmuch as an increase in the density of a display shrinks the effective field of view in the search for a target in it (Mackworth, 1976). However, Mackworth (1976) also found that searching for a target in a dense display increases fixation frequencies by only about $30 \%$ on the average, whereas searching for a target in a sparse display increases fixation durations by about $80 \%$ on the average. Thus, while it appears that increasing the difficulty of search changes both fixation durations and frequencies, it remains to be determined how stimulus factors affect these parameters of eye movements in visual search situations.

\section{REFERENCES}

BARBER, P. (1981). Visual search and number of stimuli reexamined. Psychological Bulletin, 89, 176-182.

Blough, D. (1979). Effects of the number and form of stimuli on visual search in the pigeon. Journal of Experimental Psychology: Animal Behavior, 5, 211-223.

Collin, N. G., Cowey, A., Latto, R., \& Marzi, C. (1982). The role of frontal eye fields and superior colliculi in visual search and non-visual search in rhesus monkeys. Behavioral Brain Research, 4, 177-193.

Collins, J. F., \& Eriksen, C. W. (1967). The perception of multiple simultaneously presented forms as a function of foveal spacing. Perception \& Psychophysics, 2, 369-373.

Daitch, J. M., \& Green, D. G. (1969). Contrast sensitivity of the human peripheral retina. Vision Research, 9, 947-952.

Eriksen, C. W., \& Hoffman, J. E. (1972). Temporal and spatial characteristics of selective encoding from visual displays. Perception \& Psychophysics, 12, 201-204.

EsTES, W. J. (1972). Interactions of signal and background variables in visual processing. Perception \& Psychophysics, 12, 278-286.
Gould, J. D. (1973). Eye movements during visual search and memory search. Journal of Experimental Psychology, 98, 184-195.

Gould, J. D., \& CARN, R. (1973). Visual search, complex backgrounds, mental counters, and eye movements. Perception \& Psychophysics, 14, 125-132.

LATTO, R. M. (1978a). The effects of bilateral frontal eye-field lesions on the learning of a visual search task by rhesus monkeys. Brain Research, 147, 370-376.

LATTo, R. (1978b). The effects of bilateral frontal eye-field, posterior parietal or superior colliculus lesions on visual search in the rhesus monkey. Brain Research, 146, 35-50.

Luria, S. M., \& Strauss, M. S. (1975). Eye movements during search for coded and uncoded targets. Perception \& Psychophysics, 17, 303-308.

Mack worth, N. (1976). Stimulus density limits the useful field of view. In R. A. Monty \& J. W. Senders (Eds.), Eye movements and psychological processes (pp. 307-322). Hillsdale, NJ: Erlbaum.

Neisse R, V. (1963). Decision-time without reaction time: Experiments in visual scanning. American Journal of Psychology, 76, 376-385.

NeISSER, V. (1967). Cognitive psychology. New York: AppletonCentury-Crofts.

Ne isser, V., Novick, R., \& Lazar, R. (1963). Searching for ten targets simultaneously. Perceptual and Motor Skills, 17, 955-961.

Polidora, V. J., \& Fletcher, H. J. (1964). An analysis of the importance of S-R spatial contiguity for proficient primate discrimination performance. Journal of Comparative and Physiological Psychology, 57, 224-230.

Strangert, B., \& Branstrom, L. (1975). Spatial interaction effects in letter processing. Perception \& Psychophysics, 17, 268-272.

Teichner, W. H., \& Krebs, M. J. (1974). Visual search for simple targets. Psychological Bulletin, 81, 15-28.

Treisman, A. (1982). Perceptual grouping and attention in visual search for features and for objects. Journal of Experimental Psychology: Human Perception and Performance, 8, 194-214.

Williams, L. G. (1966). Target conspicuity and visual search. Human Factors, 8, 80-92.

Wine R, B. J. (1962). Statistical principles in experimental design. New York: McGraw-Hill

(Manuscript received March 15, 1983; revision accepted for publication May 18, 1984.) 